\title{
High-Order Harmonic-Wave Generation of Ultrasonic Shear Waves
}

\author{
Mikio Fukuhara* \\ Institute for Materials Research, Tohoku University, Sendai, 980-8577, Japan
}

\begin{abstract}
The high-order harmonic-wave generation of ultrasonic shear waves has not yet been studied. We generated high-order shear harmonic waves and studied them in terms of the nonlinear wave mechanism associated with strain waves. When the shear waves propagated through highly dissipative $\mathrm{MnCu}_{20} \mathrm{Ni}_{5} \mathrm{Fe}_{2}, \mathrm{TiNi}$, and $\mathrm{Sn}-3 \mathrm{Al}$ specimens and were reflected from the far side of the specimen, we observed high-order harmonic waves up to the fifth- order of the fundamental frequency. The frequency $f$ increases linearly with the harmonic-frequency order number $n, f=a(n-1)+\mathrm{c}$. The order number coefficient $a$ increases as Poisson' s ratio increases, suggesting that the generation of high-order harmonic waves can be attributed to anharmonic solids with large elastic deformability and high transparency for shear waves.
\end{abstract}

Keywords: High-order harmonics, ultrasonic shear waves, nonlinear acoustics, Poisson’s ratio.

\section{INTRODUCTION}

Studies of the propagation of shear waves have long been of interest to researchers in the field of geophysics [1], acoustics [2], and electromagnetics [3], because shear waves provide significant phonon interaction with solids in sharp contrast to longitudinal waves [4]. The application of these studies includes technologically important areas such as nondestructive testing [5] and residual stress measurement [6]. Although it is necessary to use high-frequency shear waves for precise ultrasonic measurements, the inorganic piezoelectric resonator in the wave generation puts severe constraints on the use of high frequencies above $15 \mathrm{MHz}$ because of its brittleness. A unique way to solve this problem is the use of high-frequency harmonic waves.

It is known that a second-harmonic bulk wave is generated when a fundamental bulk wave is reflected from a linear medium to a nonlinear one [7, 8]. Indeed, Mao et al. [9] have reported that second-harmonic surface waves are generated at the interface between glass and iron. In liquids, such behavior, which has been observed by optical [10] and acoustic filter methods [11], has shed new light on the equation of state of liquids [12]. To the best of our knowledge, however, no previous research has been conducted on the generation of high-order shear harmonic waves for solids over the second degree. Our interest lies in the generation of high-order shear harmonic waves in terms of the nonlinear wave mechanism associated with strain waves. It is of great interest to generate high-frequency shear waves for industrial applications such as ultrasonic microscopy and diagnosis $[13,14]$.

Seeger and Buck [15] and Breazeale and Thompson [16] have predicted the generation of high-order harmonics when

\footnotetext{
*Address correspondence to this author at the Institute for Materials Research, Tohoku University, Sendai, 980-8577, Japan;

Tel: +81-22-215-2610; Fax: +81-22-215-2381;

E-mail: fukuhara@imr.tohoku.ac.jp
}

a sinusoidal ultrasonic wave with a sufficiently large stress amplitude is introduced into a nonlinear or anharmonic solid. Such a wave induces a distortion of the stress field. This implies a deviation from Hooke's law, which describes the relation between pressure and volume or between stress and strain in a medium. The extreme example is the formation of $\mathrm{N}$ and repeated sawtooth waves in repeated adiabatic compression and tension $[17,18]$. However, to our knowledge, since 1960, no evidence has been reported of such occurrences. A major cause for an unsuccessful result at the time would have been the usage of wave-transparent solids such as aluminum and copper [16, 19]. In this study, we investigate the possibility of the generation of high-order harmonic shear waves, utilizing anharmonic strain due to propagation of waves in nonlinear solids, and explore the effects of elastic deformability or elastic parameters.

\section{EXPERIMENTAL}

The materials used in this study are seven types of ceramics (fused quartz, silicon nitride, silicon carbide, alumina, zirconia, magnesia and graphite); nine types of metals (stainless steel (Fe-18 mass\%Cr-8 mass\% $\% \mathrm{Ni}$, SUS304), copper, aluminum, silicon, magnesium, solders (Zn-3Al, $\mathrm{Sn}-37 \mathrm{~Pb}$ ), $\mathrm{TiNi}$, and $\mathrm{MnCu}_{20} \mathrm{Ni}_{5} \mathrm{Fe}_{2}$ (M2052)); and six types of polymers (polymethyl methacrylate (PMMA), polyimide (PI), polyetherimide (PEI), polystyrene (PS), polyvinyl chlolide (PVC), and polycarbonate (PC)). Some properties of the specimens are listed in Table 1. Each specimen was in the form of a $10 \times 10 \times 10 \mathrm{~mm}$ cube. The density was determined accurately by the Archimedes method; the specimen was weighed in air and distilled water.

The apparatus and block diagram for the measurement setup are shown in Fig. (1). The gated output of a pulserreceiver was connected to an $\mathrm{A} / \mathrm{D}$ converter. The ultrasonic pulser and receiver were composed of synchronized PCI boards. The reading accuracy of the flight time for the longitudinal and shear waves was \pm 20 and 50 ps, respectively. The damping ratio and frequency of the 
Table 1. Some Properties of 22 Types of Solids Used in this Study. The Frequency Below the Names of Each Solid is the Central Frequency Used in this Study

\begin{tabular}{|c|c|c|c|c|c|c|c|c|c|c|c|}
\hline Solid & $\begin{array}{c}\text { Fused quartz } \\
2.8 \mathrm{MHz}\end{array}$ & $\begin{array}{c}\mathrm{Si}_{3} \mathrm{~N}_{4} \\
2.8 \mathrm{MHz}\end{array}$ & $\begin{array}{c}\mathrm{SiC} \\
2.6 \mathrm{MHz}\end{array}$ & $\begin{array}{c}\mathrm{Al}_{2} \mathrm{O}_{3} \\
2.8 \mathrm{MHz}\end{array}$ & $\begin{array}{c}\mathrm{ZrO}_{2} \\
2.2 \mathrm{MHz}\end{array}$ & $\begin{array}{c}\mathrm{MgO} \\
1.8 \mathrm{MHz}\end{array}$ & $\begin{array}{l}\text { Graphite } \\
2.4 \mathrm{MHz}\end{array}$ & $\begin{array}{c}\mathrm{Fe}-18 \mathrm{Ni}-8 \mathrm{Cr} \\
2.8 \mathrm{MHz}\end{array}$ & $\begin{array}{c}\mathrm{Cu} \\
2 \mathrm{MHz}\end{array}$ & $\begin{array}{c}\mathrm{Al} \\
2.6 \mathrm{MHz}\end{array}$ & $\begin{array}{c}\mathrm{Si} \\
2.8 \mathrm{MHz}\end{array}$ \\
\hline $\begin{array}{l}\text { Density } \\
\left(\mathrm{Mg} / \mathrm{m}^{3}\right)\end{array}$ & 2.220 & 3.272 & 2.981 & 3.982 & 6.050 & 3.367 & 1.799 & 7.899 & 8.936 & 2.717 & 2.331 \\
\hline $\begin{array}{l}\text { Attenuation Coefficient } \\
\text { (neper } / \mathrm{cm})\end{array}$ & 0.061 & 0.195 & 0.057 & 0.092 & 0.090 & 2.96 & 0.834 & 0.058 & 0.107 & 0.203 & 0.125 \\
\hline $\begin{array}{c}\text { Shear wave } \\
\text { Velocity }(\mathrm{m} / \mathrm{s})\end{array}$ & 3768 & 6111 & 7519 & 6246 & 3634 & 5836 & 2462 & 3128 & 2293 & 3154 & 5853 \\
\hline Solid & $\mathrm{Mg} 1.6 \mathrm{MHz}$ & $\begin{array}{c}\mathrm{Zn}-3 \mathrm{Al} \\
2.8 \mathrm{MHz}\end{array}$ & $\begin{array}{l}\mathrm{Sn}-37 \mathrm{~Pb} \\
2.4 \mathrm{MHz}\end{array}$ & $\begin{array}{c}\text { TiNi } \\
2.8 \mathrm{MHz}\end{array}$ & $\begin{array}{c}\mathrm{M} 2052 \\
2.2 \mathrm{MHz}\end{array}$ & $\begin{array}{l}\text { PMMA } \\
1.2 \mathrm{MHz}\end{array}$ & $\begin{array}{c}\mathrm{PI} \\
2.8 \mathrm{MHz}\end{array}$ & $\begin{array}{c}\text { PEI } \\
\text { 3.8 MHz }\end{array}$ & $\begin{array}{c}\text { PS } \\
2.6 \mathrm{MHz}\end{array}$ & $\begin{array}{c}\text { PVC } \\
2.6 \mathrm{MHz}\end{array}$ & $\begin{array}{c}\mathrm{PC} \\
2.8 \mathrm{MHz}\end{array}$ \\
\hline Density $\left(\mathrm{Mg} / \mathrm{m}^{3}\right)$ & 1.809 & 6.783 & 8.407 & 6.459 & 7.343 & 1.197 & 1.488 & 1.284 & 1.048 & 0.991 & 1.194 \\
\hline $\begin{array}{l}\text { Attenuation Coefficient } \\
\text { (neper/cm) }\end{array}$ & 0.280 & 0.558 & 0.415 & 0.521 & 0.455 & 1.338 & 0.521 & 0.629 & 0.608 & 2.072 & 4.062 \\
\hline $\begin{array}{l}\text { Shear wave } \\
\text { Velocity }(\mathrm{m} / \mathrm{s})\end{array}$ & 3051 & 2318 & 1464 & 2040 & 2375 & 1356 & 1417 & 1070 & 1144 & 1390 & 1113 \\
\hline
\end{tabular}

specimen were measured by use of an Ultrasonic Diagnosis and Analyzer (USH-D, Toshiba Tungaloy) at $298 \mathrm{~K}$.

Poisson' $s$ ratio was calculated from the longitudinal and shear wave velocities, $\mathrm{V}_{1}$ and $\mathrm{V}_{\mathrm{s}}$, respectively, using Elastic Parameters Measuring System (UMS-R, Toshiba Tungaloy). Broad-bandwidth shear wave PZT ceramic and PVDF polymer transducers of $0.5,1,1.6,2,5,7,10$, and $20 \mathrm{MHz}$, were used to measure the dynamic viscosity. The transducers made contact with the specimen under a pressure of $0.2 \mathrm{MPa}$ via a water-free couplant (Tungsonic oil $H$ [20]). To investigate the propagation characteristics, shear wave PZT resonators with fundamental frequencies of $1,2,3,4$, and 5 $\mathrm{MHz}$ were bonded to the highly dissipative specimen.

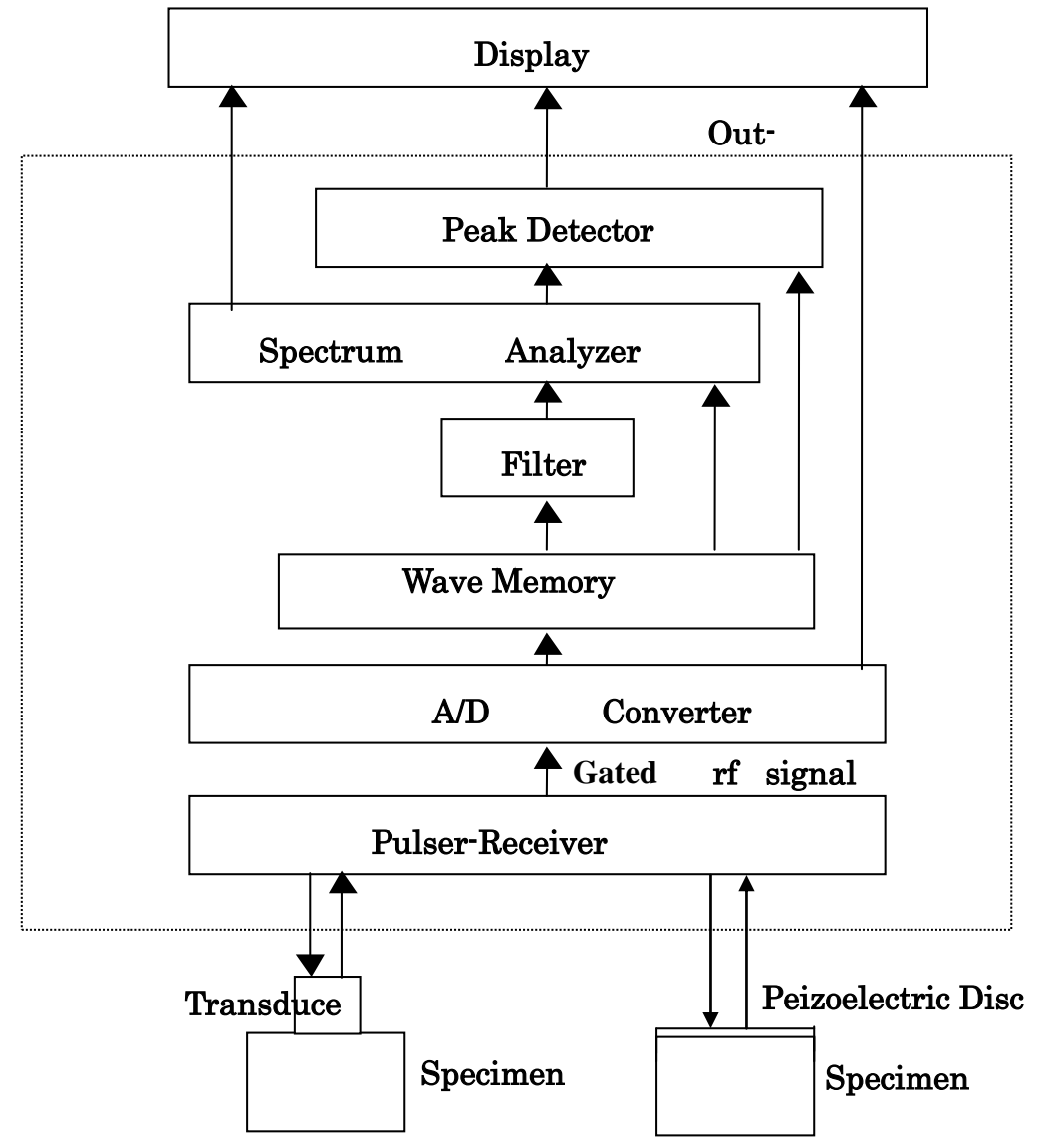

Fig. (1). Block diagram of the apparatus and setting used in this study. 
The dynamic viscosity $\eta$ is expressed by the following formula [21],

$\eta=2 \rho \alpha \mathrm{Vs}^{3} /(2 \pi \mathrm{f})^{2}$

$\alpha=\ln \left(\mathrm{A}_{1} / \mathrm{A}_{2}\right) / l$

$\mathrm{Vs}=2 l /\left(\mathrm{t}_{2}-\mathrm{t}_{1}\right)$,

where Vs, $\rho, f$, and $\alpha$ are the shear velocity, density, frequency, and attenuation coefficient, respectively, and $l$ is length of the specimen. $A_{1}$ and $A_{2}$, and $t_{1}$ and $t_{2}$ are the amplitudes and flight times of the first and second waves, respectively.

\section{RESULTS}

\subsection{Higher Dissipative Alloys for Shear Waves}

Since strain waves can be formed during propagation through dissipative solids $[15,16]$, we looked for dissipative solids with higher dynamic viscosity in terms of the nonlinear shear wave mechanism. Since waveform distortion is governed by the decrease in wavelength, we investigated the frequency dependence on the dynamic viscosity using 22 different specimens [22]. The results for 10 types of metallic, inorganic, and organic solids are shown in Fig. (2). The other 12 types of solids were eliminated because of lower dynamic viscosities. The dynamic viscosity of all specimens used in this study increases parabolically with increasing wavelength, i.e., decreasing frequency. Consequently, the highly dissipative M2052, TiNi, and Zn-3Al alloys showed the highest dynamic viscosity. The M2052 alloy has the highest damping constant [23] and TiNi is a shape memory alloy with high damping properties [24]. The solider alloy $\mathrm{Zn}-3 \mathrm{Al}$ is a unique alloy that shows creep behavior at room temperature. Thus, to utilize the acoustic distortion of the waveform, it is reasonable to use the three alloys with the highest viscosity as propagation media.

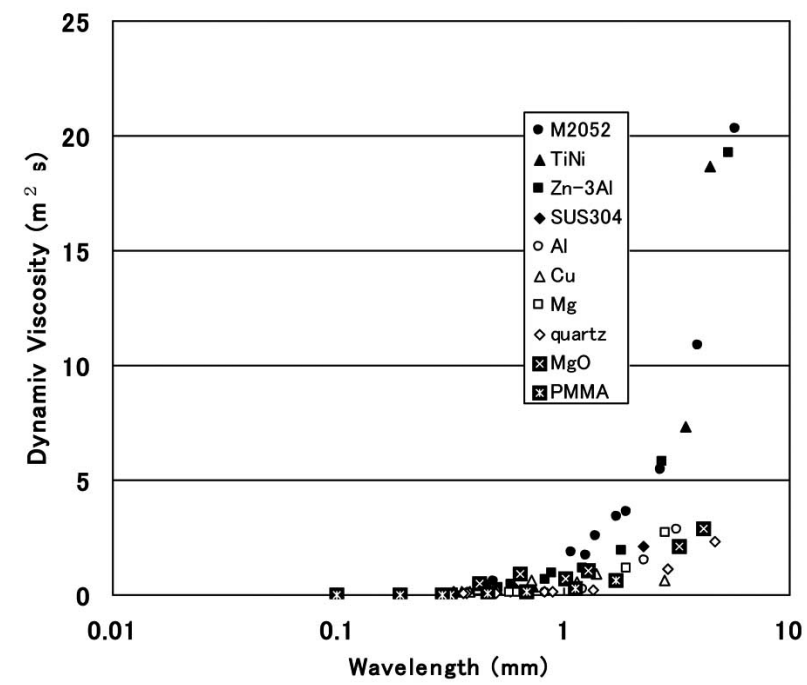

Fig. (2). Wavelength dependence of dynamic viscosity for 10 representative types of metallic, inorganic, and organic solids.

\subsection{Generation of Highest Shear Harmonic Waves}

When we use these high-viscosity alloys as propagation media for the shear waves, the emitted elastic shear waves are reflected at the far end of the alloy specimens and return back at the resonators as echoes. First, we investigated the frequency effect on the propagation characteristics of the propagation media. Fig. (3) presents the Fourier spectra of the reflected wave echoes for TiNi by using of five types of resonators with initial fundamental frequencies from 1 to 5 $\mathrm{MHz}$. The reflected wave patterns are shown in the inset for each spectrum. In contrast to the wave of $1,3,4$, and $5 \mathrm{MHz}$ that have a narrow frequency spectrum, the wave pattern with an initial frequency of $2 \mathrm{MHz}$ consists of combination of many frequencies. As a result, the resonator of $2 \mathrm{MHz}$ shows five peaks corresponding to the fifth-order harmonics of the fundamental frequency in the equency region of interest. Therefore, we used the $2 \mathrm{MHz}$ signal hereafter as the initial frequency of the resonator.

The patterns and spectra of the reflected waves for the highest dissipative alloys, M2052, TiNi, and $\mathrm{Zn}-3 \mathrm{Al}$ are shown in Fig. (4a-c), respectively, along with the results (d) for fused quartz as a reference sample. The pattern of TiNi is the most complex, while that of quartz is the simplest. Although highly transparent quartz does not generate harmonic waves, the other alloys show higher harmonics up to the fifth order.

Next we compared the amplitude of the spectrum peaks of the harmonic orders of harmonic waves in Fig. (3) as a function of the degree of harmonics. The normalized amplitude for the three dissipative alloys decreases parabolically with the order number (Fig. 5). Since the frequency of the harmonic waves does not have a fixed value, the order number dependence on frequency for these alloys is presented in Fig. (6). The relation between the frequency $f(\mathrm{MHz})$ and order number $n$ of the harmonic waves is expressed by the linear formula,

$f=a(n-1)+\mathrm{c}$,

$\mathrm{c} \fallingdotseq 2$

where $a$ and $\mathrm{c}$ are the frequency-order number coefficient and constant, respectively. The frequency variation depends on the type of dissipative alloy. The coefficient values are 4.4, 4.8, and 5.4 MHz for $\mathrm{Zn}-3 \mathrm{Al}, \mathrm{M} 2052$, and TiNi, respectively.

\subsection{Elastic Parameter}

In the previous section, with a view to exploring the generation of harmonic waves, the acoustic characteristics of harmonic waves were investigated. Since harmonic-wave generation strongly depends on the type of alloys used as the propagation media, we investigated the elastic parameter responsible for generation. From the generation mechanism of harmonics $[15,16,19]$, we may assume that the parameter must be a three-dimensional volume-non-preserving elastic function, associated with strain formation. From the elastic 


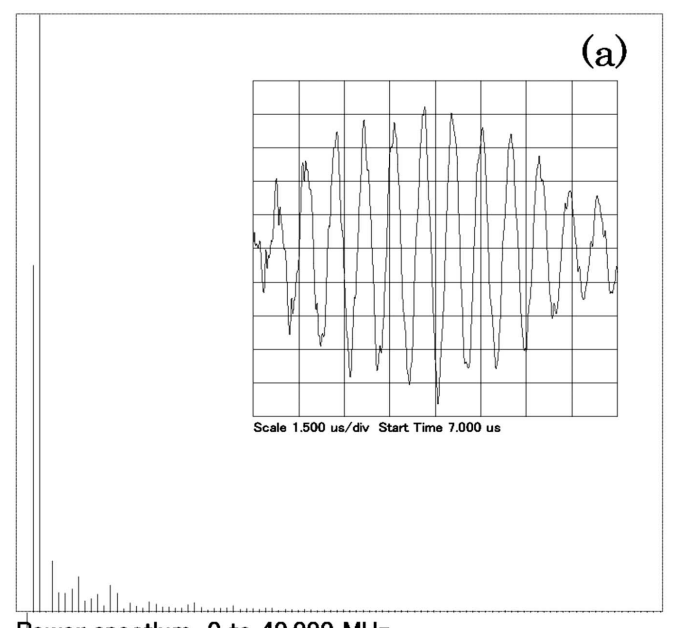

Power spectlum 0 to $40.000 \mathrm{MHz}$

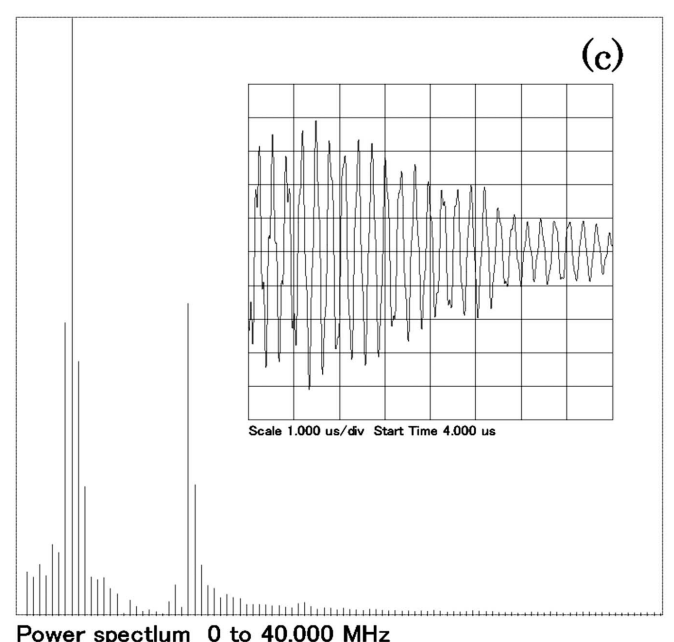

Power spectlum 0 to $40.000 \mathrm{MHz}$

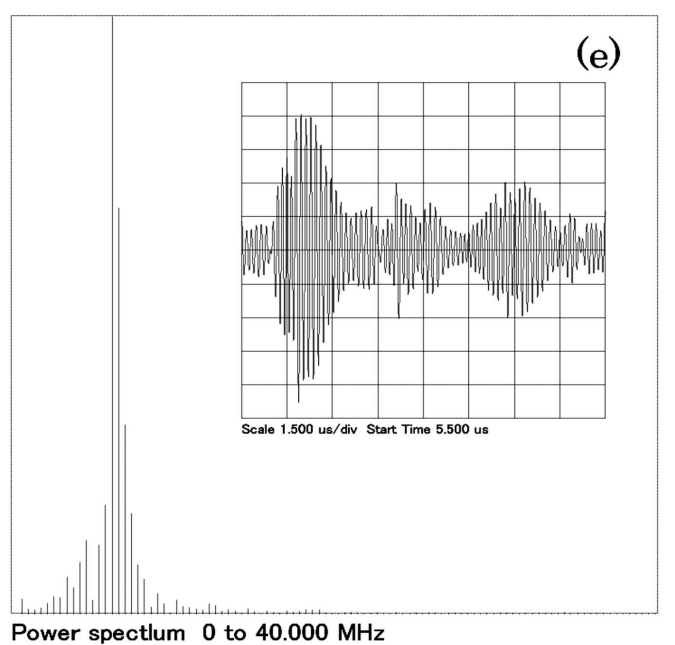

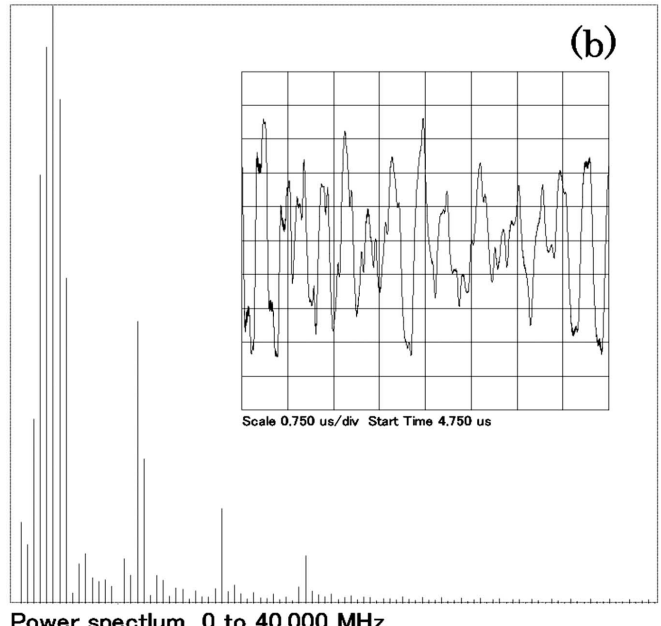

Power spectlum 0 to $40.000 \mathrm{MHz}$

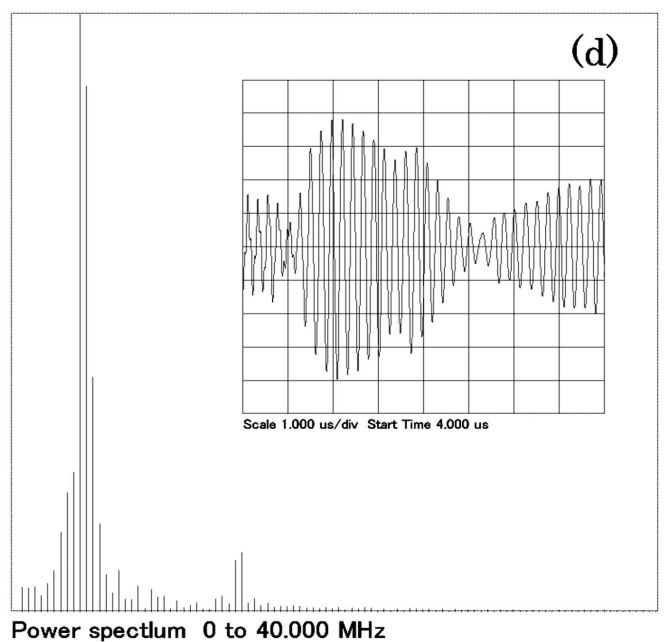

(b)

(d)

Fig. (3). Power spectra of reflected shear waves for TiNi using different resonators with frequencies of (a) $1 \mathrm{MHz},(\mathbf{b}) 2 \mathrm{MHz},(\mathbf{c}) 3 \mathrm{MHz}$, (d) $4 \mathrm{MHz}$ and (e) $5 \mathrm{MHz}$. Inset: reflected waves.

deformability viewpoint, we selected Poisson's ratio. The directional property of Poisson's ratio is designated by the quotient of lateral to longitudinal strain for all possible orientations of the coordinate system relative to the crystallographic axes. 


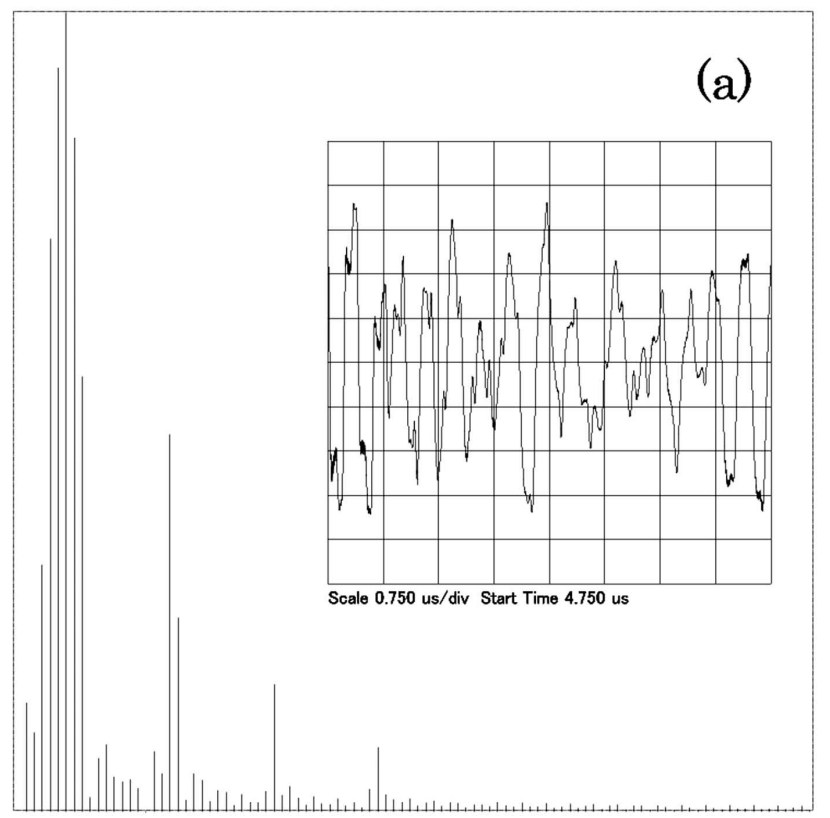

Power spectlum 0 to $40.000 \mathrm{MHz}$

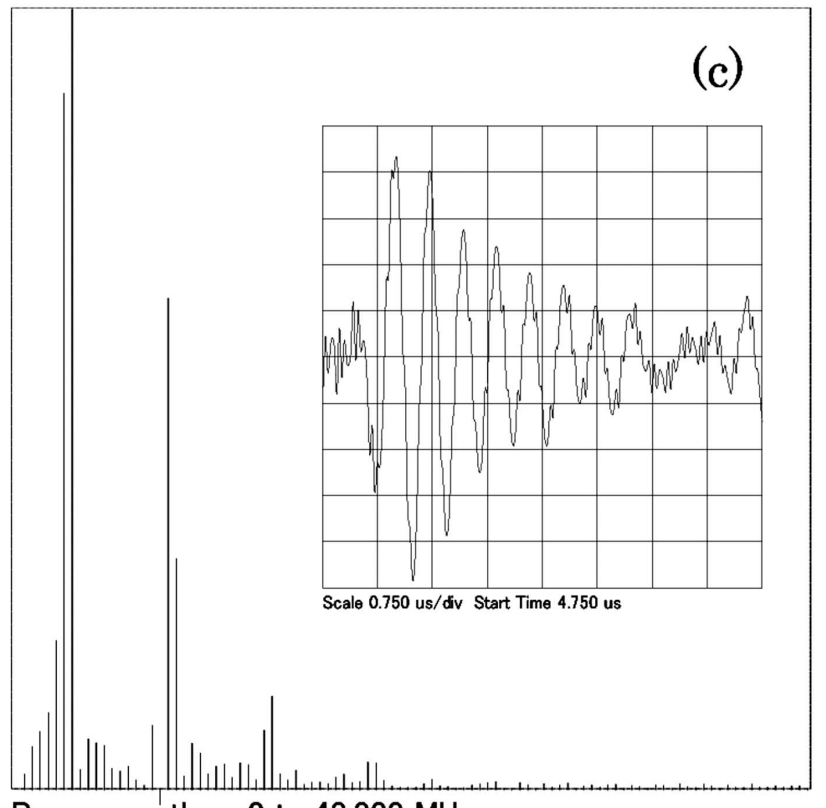

Power spectlum 0 to $40.000 \mathrm{MHz}$

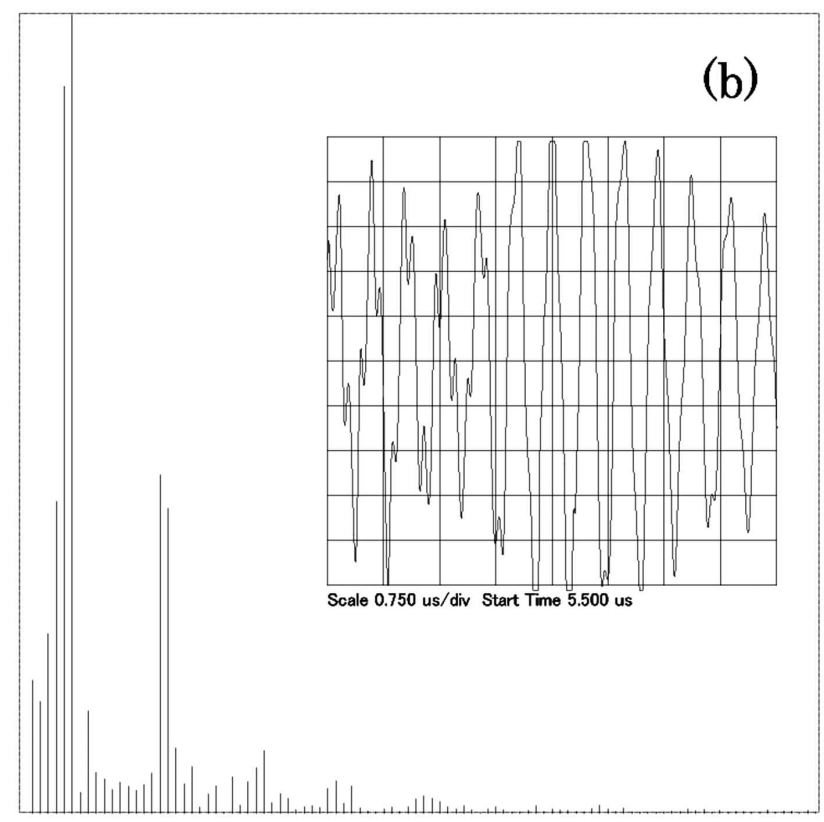

Power spectlum 0 to $40.000 \mathrm{MHz}$

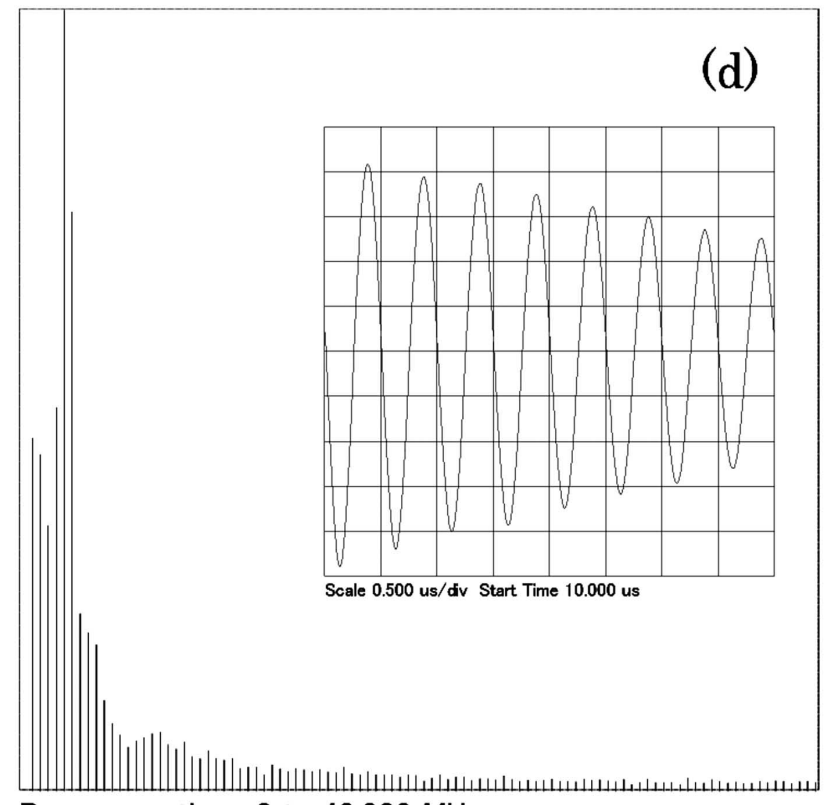

Power spectlum 0 to $40.000 \mathrm{MHz}$

Fig. (4). Power spectra of reflected shear waves; (a) TiNi, (b) M2052, (c) Sn-3Al and (d) fused quartz. Inset: reflected waves.

$v_{\mathrm{ij}}=-\frac{\mathrm{S}_{i j}}{\mathrm{~S}_{i i}}(i, j=1,2,3)$,

where $\mathrm{S}_{i j}$ stands for the elastic compliance constants.

The relation between the harmonic-frequency-order number coefficient $a$ and Poisson' $\mathrm{s}$ ratio for these alloys is shown in Fig. (7). The error allowance was within $\pm 3 \%$. The coefficient increases as Poisson's ratio increases. Thus, it is clear that the generation of harmonics is connected to their elastic deformability accompanied by three-dimensional strain.

\section{DISCUSSION}

The power spectra of the reflected shear waves for TiNi, M2052, and Zn-3Al alloys (Fig. 4), together with the wavelength dependency of the dynamic viscosity (Fig. 2) indicate that the use of metallic alloys with higher viscosity is beneficial for generating high-order harmonic shear waves. As can be seen from Fig. (3), there is a possibility that high-order harmonic waves are generated when a finite amplitude ultrasonic wave propagates adiabatically through a non-Hookean solid such as TiNi [24], because the distortion of an ultrasonic waveform through an alloy is the result of 


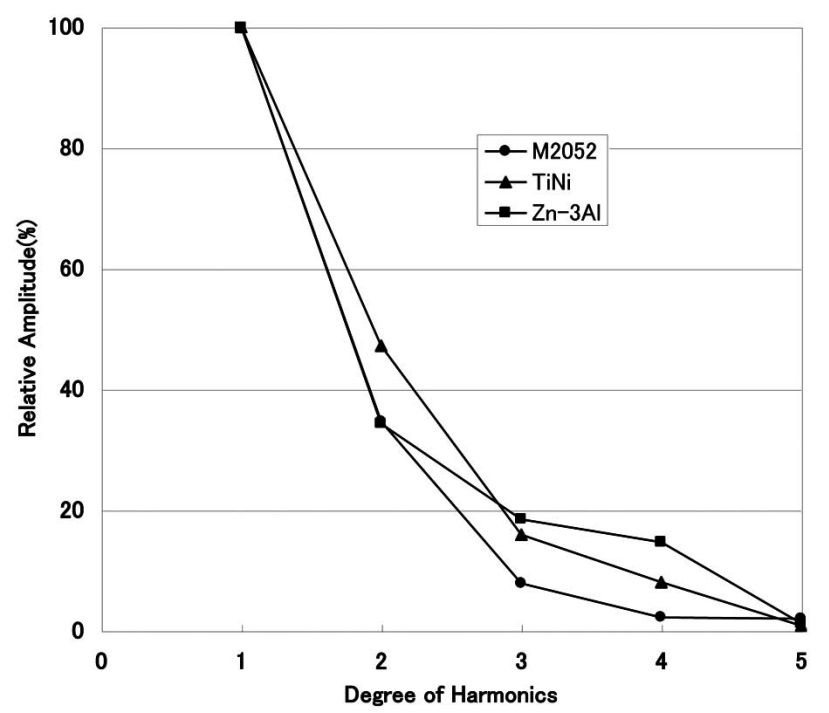

Fig. (5). Relative amplitude of harmonic waves $v s$ harmonic order number.

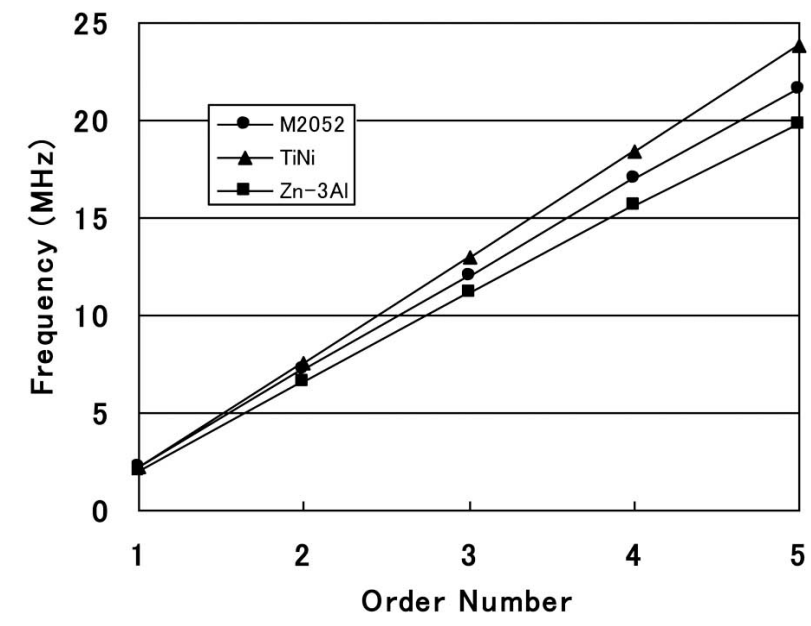

Fig. (6). Order number dependence of shear harmonic frequency of TiNi, M2052, Zn-3A1, and Sn-37Pb.

solid nonlinearity [25]. Indeed, the higher viscosity glassy alloys have sawtooth-like waveforms [26]. Nyquist diagrams of the alloys are characterized by large areas of the third and forth quadrant in the loop, suggesting an improvement of the phase delay, that is, the increment of the imaginary parts in the complex waves [27]. The distortion arises from two sources [19]: the anharmonic lattice and the movement of dislocation accompanied by nonlinear effects. The generation of high-order harmonics in this study would be due to the former because of the negligible pressure effect of the ultrasonic method.

In Fig. (7), we evaluated Poisson' $s$ ratio for the generation of higher harmonics. Since Poisson's ratio can be also expressed using $\mathrm{V}_{1}$ and $\mathrm{V}_{\mathrm{s}}$,

$v=-\frac{1}{2}\left(1-\frac{1}{\left(\frac{\mathrm{V}_{1}}{\mathrm{~V}_{\mathrm{s}}}\right)^{2}-1}\right)$, we must look for alloys with higher $\mathrm{V}_{1}$ or lower $\mathrm{V}_{\mathrm{s}}$ to generate harmonics with higher frequency. Strictly speaking, the determination of nonlinear (anharmonic) parameters has led to the calculations of combinations of third-order elastic constants [28] and certain generalized Grüneisen parameters [29]. The generalized Grüneisen parameters measure the strain dependence of the lattice vibrational frequencies.

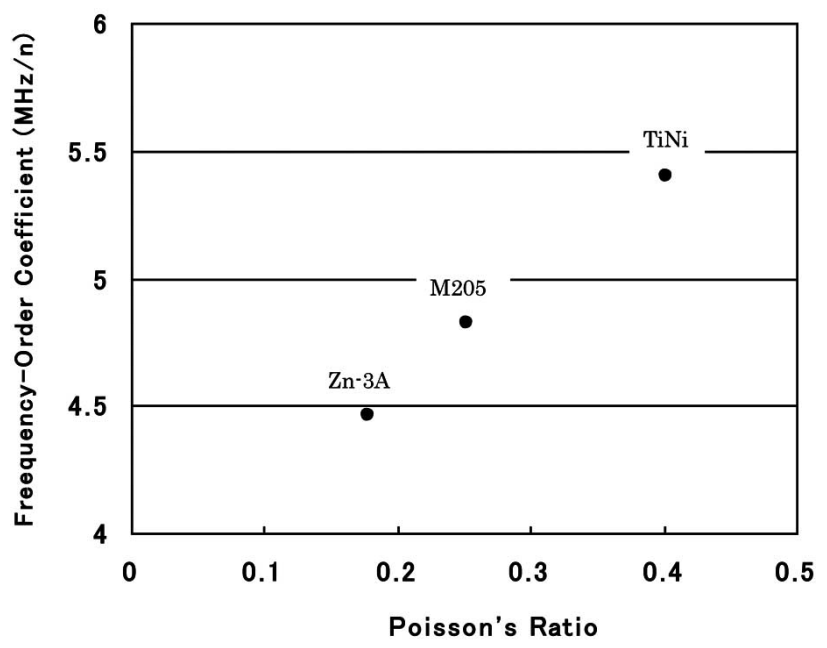

Fig. (7). Dependence of the harmonic coefficient $a(=\mathrm{d} f / \mathrm{d} n)$ on Poisson' s ratio of metallic alloys, M2052, TiNi, and Zn-3Al.

Thus, the effective generation of harmonic waves requires alloys with high elastic deformability and high transparency for shear waves. From Eq. (7), this condition is satisfied by high dissipative metallic alloys with higher $V_{1}$ and lower $\mathrm{V}_{\mathrm{s}}$. In general, inorganic ceramics have higher $\mathrm{V}_{1}$ and $\mathrm{V}_{\mathrm{s}}$, and organic polymers possess lower $\mathrm{V}_{1}$ and $\mathrm{V}_{\mathrm{s}}$. Therefore, glassy alloys with higher $\mathrm{V}_{1}$, lower $\mathrm{V}_{\mathrm{s}}$, and higher Poisson' $s$ ratio [30] would be good candidates for generation of high-order harmonic shear waves.

In this interesting field, further study is needed; attention will be placed on the generation mechanism of high-order harmonic shear waves associated with waveform deformation in highly dissipative propagation media, using computer simulation.

\section{CONCLUSIONS}

The generation of ultrasonic harmonic shear waves of orders higher than the second order has not yet been reported for solids. Following a concept based on the hypothesis of harmonic-wave generation, we first selected special alloys (M2052, TiNi, and Sn-3Al) with a nonlinear (anharmonic) lattice for the formation of ultrasonic shear strain wave by investigating the dynamic viscosity of 22 types of ceramics, metals, and polymers. For shear waves with a frequency of 2 $\mathrm{MHz}$ propagating through these alloys and reflecting from the far side of the alloy back to the resonator, these alloys generated high-order harmonic waves up to the fifth-order of the fundamental frequency. The frequency $f$ increases linearly with the order number $n(f=a(n-1)+\mathrm{c})$. The harmonic- frequency-order number coefficient $a$ increases with Poisson' s ratio increases, suggesting an increase in the elastic deformability for the formation of shear strain waves. 
The effective generation of harmonic waves requires dissipative materials with both high dynamic viscosity and high transparency for shear waves. This finding will shed light on new fields, acoustic devices, and generation mechanism.

\section{ACKNOWLEDGEMENTS}

None declared.

\section{CONFLICT OF INTEREST}

None declared.

\section{REFERENCES}

[1] Balakrishna B. Effect of dimensional orientation on ultrasonic velocities in some rocks. Proc Indian Acad Sci 1959; 49A: 318-21.

[2] Auld BA. Acoustic fields and waves in solids. New York: WileyInterscience 1973; p. 30.

[3] Vasile CF, Thompson RB. Excitation of horizontally polarized shear elastic waves by electromagnetic transducers with periodic permanent magnets. J Appl Phys 1979; 50: 2583-8.

[4] Fukuhara M. Low-temperature elastic moduli and dilational and shear Internal frictions of superconducting ceramic $\mathrm{GdBa}_{2} \mathrm{Cu}_{3} \mathrm{O}_{7-\mathrm{d}}$. Phys Rev 1994; B49: 13099-105.

[5] Fukuhara M, Kuwano Y. Propagation characteristics of $\mathrm{SH}$ ultrasonic waves through the surface depth of an isotropic medium. NDT E Int. 1998; 31: 201-10.

[6] Fortunko C, King RB. Nondestructive evaluation of planar defects in plates using low-frequency shear horizontal waves. J Appl Phys 1982; 53: 3450-8.

[7] Shui Y, Solodov I. Nonlinear properties of Rayleigh and Stoneley waves in solids. J Appl Phys 1988; 64: 6155-65.

[8] Severin FM, Solodov I. Experimental observation of acoustic demodulation in reflection from a solid-solid interface. Soviet Phys Acoust 1989; 35: 447-8

[9] Mao YW, Shui Y, Jiang W, Lu E, Wu W. Second-harmonic generation of interface waves. Appl Phys Lett 1989; 55: 2394-5.

[10] Zankel KL, Hiedemann EA. Diffraction of light by ultrasonic waves progressing with finite but moderate amplitudes in liquids. $\mathbf{J}$ Acoust Soc Am 1959; 31: 44-54.

[11] Zarembo LK, Krasil'nikov VA. Some aspects of the propagation of ultrasonic waves of finite amplitude in liquids. Soviet Phys USPEKHI 1959; 2: 580.

[12] Beyer RT. Parameter of nonlinearity in fluids. J Acoust Soc Am $1960 ; 32: 719-21$.

[13] Fukuhara M, Kuwano Y, Tsugane A, Yoshida M. Determination of thermal degradation of vulcanized rubbers using diffracted $\mathrm{SH}$ ultrasonic waves. J Polym Sci Pt. B: Polym Phys 1999 ; 37 : 497503.

[14] Fukuhara M, Kuwano Y, Matsushita M. Propagation characteristics of diffracted SH ultrasonic waves in titanium containing absorbed hydrogen. Acoust Lett 1999; 23: 60-4.

[15] Seeger VA, Buck O. Die experimentelle Ermittlung der elastischen Konstanten höherer Ordnung. Z Naturforsch 1960; 15a: 1056-67.

[16] Breazeale MA, Thompson DO. Finite-amplitude ultrasonic waves in aluminum. Appl Phys Lett 1963; 3: 77-8.

[17] Nakamura A. Takeuchi R, Oie S. Nonlinear attenuation of an $\mathrm{N}$ wave propagating in a tube, including dissipation due to wall effects. J Acoust Soc Am 1978; 63: 346-52.

[18] Nakamura A. Comparison of energy dissipation between $\mathrm{N}$ and repeated sawtooth waves with finite amplitude. J Acoust Soc Jpn(E) 1983; $4: 1-4$.

[19] Breazeale MA, Ford J. Ultrasonic studies of the nonlinear behavior of solids. J Appl Phys 1965; 36: 3486-90.

[20] Fukuhara M, Tsubouchi T. Naphthenic hydrocarbon oils transmissible for transverse waves. Chem Phys Lett 2003; 371: 184-8.

[21] Fukuhara M. Acoustic characteristics of botanical leaves using ultrasonic transmission waves. Plant Science 2002; 162: 521-8.

[22] Nakamura A, Otozai T, Takeuchi R. Propagation of finite amplitude wave through circular pipe with discontinuity cross sections. J Acoust Soc Jpn(E) 1980; 2: 141-9.

[23] Fukuhara M, Yin F, Kawahara K. Acoustic characteristics of high damping $\mathrm{Mn}_{73} \mathrm{Cu}_{20} \mathrm{Ni}_{5} \mathrm{Fe}_{2}$ alloy. Phys Status Solidi(A) 2004; 201: 454-8.

[24] Fukuhara M, Yagi M, Matsuo A. Temperature dependence of elastic parameters and internal frictions for TiNi alloy. Phys Rev 2002; B65: 224210.

[25] Cantrell JH, Yost WT. Rev. Progress in quantitative nondestructive evaluation. In: Thompson DO, Chementi DE, Eds. New York: Prenum Press 1990; Vol. 9: p. 1661

[26] Fukuhara $M$, Zhang $W$, Louzguine-Luzgin DV. Inoue A. Ultrasonic attenuation properties of glassy alloys in views of complex viscoelasticity. Appl Phys Lett 2007; $90: 131902$.

[27] Fukuhara M, Kawashima A, Zhang W, Inoue A. Low temperature dependence of elastic parameters and internal frictions for glassy alloy $\mathrm{Cu}_{45} \mathrm{Zr}_{45} \mathrm{Al}_{5} \mathrm{Ag}_{5}$. J Appl Phys 2008; 103: 013503.

[28] Yost WT, Cantell JH, Breazeale MA. Ultrasonic nonlinearity parameters and third-order elastic constants of copper between 300 and $3^{\circ} \mathrm{K}$. J Appl Phys 1981; 52: 126-8.

[29] Cantell Jr JH. Generalized Grüneisen tensor from solid nonlinearity parameters. Phys Rev 1980; B21: 4191-5.

[30] Fukuhara M, Zhang W, Inoue A. Ultrasonic properties of glassy alloys in terms of complex wave elasticity. Phys Status Soldi 2007; 204: 3116-20. 